\title{
DETECCIÓN DE ALTERACIONES DEL DESARROLLO INFANTIL EN NIÑOS CON TRASTORNO DE DÉFICIT ATENCIONAL CON HIPERACTIVIDAD EN PUNTA ARENAS: EXPLORANDO LA VISIÓN DE LOS PADRES
}

\section{DETECTION OF CHANGES IN CHILD DEVELOPMENT CHILDREN WITH ATTENTION DEFICIT HIPERACTIVITY DISORDER IN PUNTA ARENAS: EXPLORING THE VISION OF PARENTS}

\author{
Daniela Mandiola G. ${ }^{1}$ Karina Oyarzo M. ${ }^{2}$ Nancy Vásquez L. ${ }^{3}$
}

\begin{abstract}
RESUMEN
Esta investigación indagó los procesos de detección de alteraciones del desarrollo infantil en niños con Trastorno de Déficit Atencional e Hiperactividad (TDAH) en Punta Arenas, desde la perspectiva de los padres, debido a la escasa investigación en la región con respecto al tema, a su relevancia para la profesión de Terapia Ocupacional y a la repercusión que tiene esta problemática en políticas nacionales y mundiales de salud.

Su metodología es cualitativa, con un diseño basado en el interaccionismo simbólico. Los participantes de la investigación fueron padres y/o madres de niños con diagnóstico de TDAH. Para la recolección de datos se utilizó la entrevista semi- estructurada en profundidad, los que posteriormente fueron analizados desde la Teoría Fundamentada.

Se identifica que los padres tienen conocimiento general sobre el desarrollo infantil de sus hijos, aunque desconocen los signos de alerta del desarrollo psicomotor. Además, manifiestan falta de información y orientación de parte de los profesionales de redes asistenciales de salud y educación. Refieren la importancia de realizar esta detección antes de los seis años.

Se concluye que el proceso de detección de alteraciones en el desarrollo infantil es tardío y extenso para las familias, pese a los distintos programas de salud y educación existentes.
\end{abstract}

Palabras claves: Trastorno de Déficit Atencional e Hiperactividad, Terapia Ocupacional, Desarrollo Infantil.

\begin{abstract}
This research investigated the processes of diagnosis of child development in children with Attention Deficit Hyperactivity Disorder (ADHD) in Punta Arenas, from the perspective of parents, due to the limited research in the region on the issue, in relevance to the profession of occupational therapy and the impact that has this problem in national policies and global health. The methodology is qualitative, with a design based on symbolic interactionism. The research participants were parents and / or mothers of children diagnosed with ADHD. Data collection was semi-structured analyzed from the Grounded Theory. We showed that the parents have general knowledge of child development of their children, but not known the warning signs of psychomotor development. Furthermore, they have shown a lack of information and guidance from professionals in health care networks and education. For parents it is important to observe the warning signs of child development before age six. We conclude that the process of diagnosis of child development is delayed for families, despite the various health and education programs available.
\end{abstract}

Keywords: Attention Deficit Hyperactivity Disorder, Occupational Therapy, Child Development.

${ }^{1}$ Licenciada en Ciencia de la Ocupación, Terapia Ocupacional, Universidad de Magallanes, Punta Arenas,mandiola.daniela@gmail.com, Tel.(61) 224001

${ }^{2}$ Licenciada en Ciencia de la Ocupación, Terapia Ocupacional, Universidad de Magallanes, Punta Arenas, kry_115@hotmail.com, Tel.(61) 212097

${ }^{3}$ Licenciada en Ciencia de la Ocupación, Terapia Ocupacional, Universidad de Magallanes, Punta Arenas, vivivasquezl@ hotmail.com, Tel.(61) 212610 


\section{INTRODUCCIÓN}

El Trastorno por Déficit Atencional con Hiperactividad (TDAH) es el diagnóstico neuropsiquiátrico infantil de mayor prevalencia a nivel mundial, situándose entre un $3 \%$ y un $7 \%$ de los niños en edad escolar (López-Ibor 2002). En España, presenta una alta demanda asistencial, ocupando el primer lugar en consultas atendidas, correspondiendo a un 86\% (Círez, I., Pérez, B. y López, S , 2009). En Chile, se ha encontrando que uno de cada 80 a 100 escolares es diagnosticado en los servicios de atención primaria (Lavados P., Gómez V., Sawada M., Chomalí M., Álvarez M. 2003). La cuidad de Punta Arenas no está ajena a esta problemática, presentando en el año 2011 cifras que bordean los 330 casos diagnosticados, de los cuales $42 \%$ son menores de 9 años, el $45 \%$ se encuentra en el tramo de 10 a 14 años y un 13\% de 15 a 19 años (Servicio de Salud Magallanes, 2011).

Como lo señala el DSM-IV-TR, en sus criterios para el diagnóstico, los síntomas de hiperactividad/impulsividad deben haber estado presentes antes de los 7 años de edad (López-Ibor, 2002), sugiriendo esperar hasta la edad escolar para entregar un diagnóstico más preciso por parte del equipo médico. Sin embargo, hoy en día los padres cumplen un papel de suma importancia en el contexto de la vigilancia del desarrollo de sus hijos. En general, existe consenso en la literatura de que, en su mayoría, los padres son buenos observadores y detectores certeros de las deficiencias observadas en sus hijos, cuya opinión tiene un buen valor predictivo para la detección de alteraciones del desarrollo (Figueiras, A. 2006).

De aquí, radica la importancia de indagar en los procesos de detección de alteraciones del desarrollo infantil en niños con TDAH en Punta Arenas, desde la perspectiva de los padres, debido a la escasa investigación existente en la región con respecto al tema, a su relevancia para la profesión de Terapia Ocupacional y a la repercusión que tiene esta problemática en las políticas nacionales de salud y también a nivel mundial.

Esta investigación aporta nuevos antecedentes en el proceso de la detección temprana, desde la perspectiva de las propias personas involucradas. Se espera, de esta manera, aportar con bases científicas a la mejoría en la atención primaria en la ciudad de Punta Arenas, y con ello generar nuevos campos laborales dirigidos a la prevención y promoción de la salud, a través de las prácticas de la Terapia Ocupacional.

\section{METODO}

Este estudio se abordó desde un enfoque cualitativo. Bajo este punto de vista, esta metodología permite conocer la perspectiva que tienen los padres sobre los procesos de detección temprana de signos de alerta de niños con TDAH, "viendo el escenario y a las personas desde una perspectiva holística donde el ser humano y su entorno no son reducidos a variables sino considerados como un todo" (Figueiras, A. C Figueiras, A. 1997). 
El lugar donde se observó el fenómeno fue en los domicilios particulares de cada uno de los participantes, los cuales fueron captados a través de las redes de atención primaria en la ciudad de Punta Arenas.

Los participantes de esta investigación cumplieron con los siguientes Criterios Muestrales:

- Ser padre o madre de un niño(a) diagnosticado(a) con TDAH

- Ser padre o madre de un niño (a) menor de11años.

- Que el diagnóstico del niño (a) se haya realizado hace 3 añoso menos.

- Aceptar participar voluntariamente en la investigación (firmando Consentimiento Informado).

Cuadro $\mathrm{N}^{\circ}$ 1: Caracterización de los participantes investigados

\begin{tabular}{|l|l|l|l|}
\hline \multicolumn{1}{|c|}{$\mathbf{N}^{\circ}$} & \multicolumn{1}{|c|}{ Rol } & $\begin{array}{c}\text { Edad hijo/a con } \\
\text { TDAH }\end{array}$ & $\begin{array}{c}\text { Edad del } \\
\text { Diagnóstico }\end{array}$ \\
\hline Entrevista 1 & Madre & 8 & 6 \\
\hline Entrevista 2 & Madre & 7 & 6 \\
\hline Entrevista 3 & Madre & 11 & 8 \\
\hline Entrevista 4 & Madre y padre & 6 & 6 \\
\hline Entrevista 5 & Madre & 8 & 8 \\
\hline Entrevista 6 & Madre & 9 & 7 \\
\hline
\end{tabular}

Fuente: Autoras

La totalidad de los menores recibieron el diagnóstico en el período de educación formal básica.

La técnica de recolección de datos que resultó más adecuada para acercarse al objetivo de estudio de esta investigación fue la entrevista en profundidad semiestructurada. En la elaboración y aplicación de entrevista se levantaron los siguientes ejes temáticos:

- Conocimiento previo acerca de signos de alerta temprana en el desarrollo del niño.

- Características del proceso o etapas desde la detección de las alteraciones hasta la búsqueda de ayuda

- Forma ideal de la detección de los signos de alerta.

El modelo de análisis de datos que se adoptó en esta investigación es la Teoría Fundamentada. Esta teoría da la posibilidad de reflexionar sobre las experiencias aportadas por los padres, también permite reflexionar, generar una teoría a través de un modelo explicativo-relacional, tener creatividad y un pensamiento crítico sobre la evidencia entregada (Goetz, J. Lecompte, M. 1988).

Para realizar esta investigación se respetaron los derechos de los participantes. Con este fin, todas las personas ejercieron el derecho a hacer uso de su autonomía, en forma libre y espontánea. Firmaron un consentimiento informado, documento a través del cual expresaron su voluntad de participar en la investigación, manifestaron tener conocimiento absoluto sobre la investigación, de haber sido informados del propósito de la misma, así como de los objetivos y sobre el uso de la información recolectada, exclusivamente para 
fines de la investigación. Así también, se les aseguraba la máxima confidencialidad y anonimato. Por último, ejercieron el derecho de no participar, de retirase en cualquier momento, o de negarse a contestar algún tipo de pregunta si lo consideraba inoportuno.

\section{RESULTADOS}

Los resultados se presentan en base a cada eje temático estudiado.

$\underline{\text { 1. Conocimiento previo de los padres sobre los signos de alerta en el desarrollo }}$

\subsection{Conocimiento general sobre desarrollo infantil}

De acuerdo a la retrospección en relación al desarrollo infantil de sus hijos, previo a la realización del diagnóstico, los padres expresan que identificaron hitos generales y básicos del desarrollo tales como la adquisición del lenguaje, bipedestación y sedestación. Los entrevistados manifiestan "Como al año ocho mes habla por ejemplo, antes del año y medio pa' caminar, para sentarse si me lo sé todo como a los seis a ocho meses...” (E3)

\subsection{Conocimiento de periodos críticos en el desarrollo}

Muchos de los relatos refieren no conocer sobre periodos críticos sobre el desarrollo psicomotor de un niño. Ellos comentan: "Nada nada, no" (E1); "Nooo la verdad es que no me había fijado, de hablar sí, pero de caminar no, de que si no caminaba al año o caminaba después del año, si no hablaba claro que si, en parte si como que uno no toma mucho en cuenta esas cosas, como que omite esas partes, pero de hablar de esas partes como que me recuerdo que me hablaban" (E2)

\section{$\underline{1.3 \text { Formas de adquisición del conocimiento }}$}

Según refieren los entrevistados principalmente se adquiere el conocimiento a través de la experiencia y la comparación en relación a otros niños en la misma etapa del desarrollo (hijos, familia, amigos). De esta manera lo explicitan: "Sí, porque cuando él era guagüita él no dormía, dormía muy pocas horas concentrado en el sueño, y se despertaba y lloraba ççes decir dormía, si una guagua tiene que dormir como 8 horas 9 o 10 horas él dormía 4, hasta como el año, pero siempre fue así como que saben en donde nosotros notamos bien esos puntos, nosotros tenemos dos hijos más grandes y fui distinto muy distinto el pasar con ellos y con él, entonces uno igual percibe la diferencia” (E4).

En resumen, los principales hitos del desarrollo general infantil que los padres observan son hitos básicos que habitualmente se obtienen de redes primarias de salud. La gran mayoría de ellos refiere no tener conocimiento acerca de los signos de alerta dentro del desarrollo psicomotor en el niño. La adquisición del conocimiento sobre alteración del desarrollo se obtiene principalmente a través de experiencias y comparaciones de crianzas previas y/o terceras personas. 
2. Proceso vivido desde la detección hasta la búsqueda de ayuda.

\subsection{Signos y síntomas mayormente identificados:}

En relación a los síntomas y conductas que llamaron la atención de los padres para que estos decidieran buscar ayuda, es posible distinguir 3 tipos, los que coinciden con los criterios diagnósticos de TDAH establecidos por el Manual de Diagnóstico DSM-IV. Los padres reconocieron algunas conductas teóricamente asociables a estos criterios: hiperactividad, impulsividad y falta de atención. De estos, los padres entrevistados reconocen con mayor énfasis el primero de ellos, la hiperactividad: "pa mí no es normal en un niño de esa edad, yo sé que todo niño hace rabieta como todos, pero no como lo que hace" (E1); “...era demasiado, o sea, 7 meses inquieto que le daban los nervios era muy claro, de primera causa risa las cosas que hace, pero ya al año que ya son inquietos, después a los 3 años ya se notaba que era inquieto" (E2).

\subsection{Proceso vivido desde la detección hasta la búsqueda de ayuda}

Este proceso fue experimentado como un largo e incierto recorrido, ya que la gran mayoría de los entrevistados mencionaban: “... lo llevé a la fonoaudióloga y la fonoaudióloga me dijo que tenía problemas del lenguaje y que mejor había que colocarlo en una escuela de lenguaje que mejor había que colocarlo en un colegio de lenguaje y ella mismo me ayudó a buscar un colegio y ahí lo matriculé y ahí la tía que tenía aparte de ser profesora diferencial era psicopedagoga igual y ella me dijo que tenía algunos problemas que aparte del lenguaje era hiperactivo y que porqué no consultaba con un neurólogo y ahí yo lo lleve a una neuróloga en Talcahuano y la neuróloga me recetó algunas gotitas para bajar la revoluciones y me acuerdo que le daba como 3 gotitas..." (E2).

El tiempo transcurrido para realizar el diagnóstico puede llevar mucho tiempo, retrasando el tratamiento y con ello la posibilidad latente de que se presenten otras patologías asociadas al TDAH. "Bueno fue cuando terminó el jardín y empezó. Él hizo el pre kínder y primero básico, porque de kínder a primero como que fue más fue aumentando más, o sea, ahí la tía reclamaba todos los días y la profesora igual cuando entró a primero y ahí fue donde lo empecé a llevar" (E3).

\section{$\underline{2.3 \text { Principal lugar de búsqueda de ayuda }}$}

Según refieren los entrevistados, al notar ciertas alteraciones en el desempeño de los niños, acudieron en una primera instancia a los consultorios de atención primaria, los que principalmente fueron una manera de buscar ayuda y orientación profesional: “...neuróloga profesional, a una neuróloga, sí bueno, ahí le hicieron un test, lo hicieron escribir qué sé yo, y ahí, bueno, ella me dijo sí de que sí efectivamente tenía una hiperactividad asociada al déficit atencional..." (E5); "al consultorio, a la doctora, la doctora que lo veía y que siempre lo ha visto desde que nació" (E4). 
En definitiva, dentro del proceso vivido hasta la búsqueda de ayuda, fueron identificados claramente por los padres, los 3 síntomas básicos del TDAH: hiperactividad, déficit atencional e impulsividad. Este proceso transcurrió de manera larga e incierta debido a la falta de información y orientación de los profesionales existentes dentro de redes asistenciales más cercanas. Este transcurso por lo general duraba como mínimo un año, yendo a consultar primeramente las redes de atención primaria, en especial los consultorios correspondientes a sus domicilios.

\section{Tipo ideal de detección de signos de alerta.}

\section{$\underline{3.1 \text { Procedimiento ideal para llegar a la detección de signos de alerta. }}$}

La detección temprana es señalada por los entrevistados como un proceso fundamental para el diagnóstico a tiempo y evitar la demora en el tratamiento, y de esta manera, poder atenuar los síntomas característicos del trastorno. "Ayudar a prevenir que esas conductas se amplien, no cierto, como ir trabajando con eso para evitar el diagnosticar o ponerlos a tratamiento cuando ya está todo hecho en vez que si quizás de chiquitito se plantea como un plan de trabajo como de evitar un poco de prevenir de ir compensando..." (E6).

A su vez, plantean que el diagnóstico debería realizarse antes de los 7 años, edad diagnóstica según criterios de DSM-IV. "Me hubiera gustado enterarme antes cuando estaba más chico para haber tenido ayuda, cuando ya tenía sus 4 años” (E2). “...Creo que los tres años es lo ideal, tres a cuatro años, cuando están en la pre-básica, yo creo que ahí es donde debería empezar todo el tratamiento..." (E4).

Refieren que debiese existir una mayor promoción y prevención de la salud por parte de profesionales, en cuanto a la entrega de información sobre alteraciones en el desarrollo psicomotor de los niños, y signos de alerta que podrían transformase en futuras patologías y comorbilidad. "Si en el jardín quizás si me hubiesen dicho que él iba no sé nunca me lo dijeron que él iba más atrasado que sus compañeros quizás yo hubiese tomado otras medidas" (E5).

En síntesis, los padres identifican claramente la importancia de realizar un diagnóstico temprano con el fin de iniciar el tratamiento lo más precoz posible y evitar su demora. Apuntan a que el diagnóstico debiera ser en etapas pre-escolares y que debería existir mayor conocimiento y manejo por parte de los profesionales en éstas áreas, de manera de otorgar una mayor orientación a las familias Además, manifiestan la necesidad de la existencia de mayor número de profesionales ligados a la detección temprana.

\section{DISCUSIÓN}

La principal conclusión que surge de este trabajo es que los procesos de detección de alteraciones del desarrollo infantil en niños con TDAH en Punta Arenas desde la perspectiva de este grupo de padres, resultaron ser procesos tardíos y extensos para las familias. Este hecho es atribuido a la escasa información que les proporcionaron las redes de Atención Primaria de la región con respecto a las manifestaciones clínicas del cuadro de 
sus hijos y a la demora en la derivación oportuna hacia profesionales de salud y educación ligados a la estimulación del desarrollo infantil del niño.

También se concluye que, a pesar de los planes impulsados por el Ministerio de Salud y Educación, para los padres la detección de este trastorno en sus hijos no es suficientemente oportuna, ya que la demora del diagnóstico retrasa la entrega de un tratamiento pertinente, aumentando su repercusión en las distintas esferas en las que se desenvuelve el niño, aumentando además las probabilidades de que esta patología se asocie a otras.

Según lo referido por los padres, la demora del diagnóstico se podría deber a la falta de información y/o conocimiento de profesionales ligados a la enseñanza de educación prebásica y básica, sobre patologías del área infantil y al trabajo en prevención propios para disminuir síntomas y signos de alteraciones del desarrollo infantil. Por ende, creen que difícilmente podrían diseñar y poner en práctica estrategias que favorezcan la detección temprana de niños que pudieran poseer TDAH antes que se lleguen a establecer mayores dificultades asociadas: trastornos del aprendizaje, problemas conductuales, ansiedad, baja autoestima, entre otras.

En este sentido, los países miembros de la Organización de las Naciones Unidas (ONU) como Chile, han establecido un compromiso mundial para mejorar la calidad de vida infantil, lo que se ha consignado en los denominados "Objetivos del Milenio", donde se ha establecido la importancia y compromiso internacional por garantizar a los niños un adecuado proceso de crecimiento y desarrollo como una de las estrategias para lograr dicho objetivo (Kofi, A 2005).

De acuerdo al compromiso establecido por la ONU, la realidad regional en salud en relación a la prevención y promoción en la región de Magallanes y Antártica Chilena, específicamente la ciudad de Punta Arenas, se caracteriza por la falta de información existente hacia los padres en los consultorios y establecimientos de atención primaria. Al no existir suficiente información, los padres desconocen los signos de alerta o manifestaciones que llamen la atención como para acudir en busca de atención médica de manera oportuna, desencadenando en un retraso en la atención del menor y, por ende, un diagnóstico tardío.

Por otra parte, el largo tiempo que trascurre entre los primeros síntomas y el diagnóstico del TDAH, se debe a lo difícil que resulta realizar un diagnóstico diferencial del TDAH por parte de los especialistas del área de la neuropsiquiatría, ya que se deben realizar varias sesiones por parte del especialista para concluir con el diagnóstico, el que por lo general, es por exclusión, debido a que el Trastorno por Déficit Atencional frecuentemente no se presenta sólo, sino que existen otros trastornos asociados (Vaquerizo-Madrid, J 2005).

El programa de salud del niño/a tiene como finalidad contribuir con estrategias de promoción al desarrollo integral y armónico del niño/a menor de diez años, a través de actividades de fomento, prevención, recuperación de la salud y rehabilitación del daño, que permitan la plena expresión de su potencial genético y una mejor calidad de vida. (Grath, L 2009) 
Que las consultas sean realizadas por iniciativa propia de los padres y no sugeridas dentro del control de salud del niño preescolar que el Ministerio de Salud realiza, demuestra que falta potenciar y difundir, aún más, los programas de prevención y promoción de salud que se han creado, necesitando posiblemente, capacitar con mayor frecuencia a los profesionales encargados de dar a conocer estos programas, los cuales, además, deben saber identificar los signos de alerta del desarrollo para luego realizar una atención temprana, ya que para los padres resulta importante que exista un diagnóstico temprano para iniciar de esta manera un tratamiento oportuno y eficiente.

Por otra parte, existen diversas evaluaciones que pesquisan trastornos del desarrollo infantil y que los pediatras deben realizar en los controles de supervisión de salud. Pese a la existencia de estas evaluaciones, el diagnóstico del TDAH se da con mayor frecuencia en niños entre los 6 a 14 años (escolares), debiendo ser detectados con anterioridad signos de alerta del desarrollo psicomotor, antes de que el niño ingrese a la etapa escolar (9), evitando de esta manera los trastornos asociados y dificultades en el desempeño de los niños en los distintos contextos en que se desenvuelven. Si bien el DSM-IV propone como referencia, la edad de 7 años como la edad apropiada para realizar un diagnóstico de esta patología, en la infancia y etapas pre- escolares ya existen signos de alerta, y manifestaciones clínicas observadas por los padres que coinciden con la clínica propuesta por investigadores, que contribuirían a un diagnóstico temprano, entre ellas se encuentran; alteración de patrones del sueño durante la lactancia, bebé incansable, demandante, llorón y movedizo, niño desobediente, gran tendencia a sufrir accidentes, niño intranquilo y con tendencia a actuar antes de pensar, dificultad de seguir ordenes de la educadora de párvulo, niños presentan agresividad con compañeros. Además estas manifestaciones tempranas del trastorno podrían ser pesquisadas, a través de exámenes de neuroimagen, ya que existen diferentes teorías que explican el origen del trastorno, y se considera la causa biológica, orgánica, como principal responsable, entendiéndose ésta como un desequilibrio químico en las áreas cerebrales involucradas en la atención y el movimiento (Grath, L 2009)

La realidad regional en educación en la región de Magallanes evidencia que el TDAH es uno de los problemas más frecuentes en niños, niñas y adolescentes, lo que en parte evidencia que hoy existe mayor conciencia sobre esta enfermedad por parte de padres, docentes y profesionales del área de la salud. Pese a esto, los profesionales relacionados con el desarrollo infantil reconocen los síntomas y signos de este trastorno básicamente en la etapa escolar, cuando el menor se ve sometido a exigencias mayores y a un clima más difícil de manejar, repercutiendo en el proceso de enseñanza, aprendizaje y traduciéndose en ocasiones en trastornos emocionales y en otros trastornos asociados al TDAH como es el caso del trastorno disocial o trastornos del aprendizaje, cada vez más frecuentes como lo indican diversas estadísticas (Grath, L 2009).

Para muchos profesionales en especial del área de educación, resulta difícil realizar una detección temprana en etapas pre-escolares, lo que podría deberse a la falta de conocimiento sobre los signos evolutivos del TDAH que comienzan en etapas tempranas del desarrollo. Muchas veces esto conlleva a una deficiente orientación hacia los padres con respecto a los pasos a seguir o bien a cómo prevenir que la sintomatología del TDAH afecte 
el desempeño del menor en todas las áreas del desarrollo, emocional, cognitiva, afectiva, social y sensorial. En relación a esto, la UNICEF (2006) menciona que:

200 millones de niños menores de cinco años en el mundo, no están cumpliendo con su potencial de desarrollo, debido a que los ambientes donde viven no les proporcionan suficiente estimulación y enseñanza, los niños se desarrollan más lentamente, o no desarrollan el pensamiento crítico y habilidades de aprendizaje. Esta limitación en el desarrollo temprano, contribuye al ingreso tardío a la escuela, bajo rendimiento escolar, y en última instancia, las limitaciones para el éxito posterior en la vida (UNICEF 2006)

En relación a la dificultad para detectar los signos de alerta o bien en el retraso en el desarrollo psicomotor, es que el proceso vivido por los padres y niños, desde el conocimiento de los primeros síntomas y signos del TDAH hasta la búsqueda de ayuda profesional transcurre un largo tiempo, siendo este no menor a un año, tiempo en el cual los padres expresan vivir con inseguridad y preocupación sobre todo porque los menores son en su mayoría discriminados por sus propios pares y en ocasiones por los propios docentes a cargo, además de presentar una disminución en su autoestima producto de no sentirse competente frete a las demandas exigidas.

Existe además, un desconocimiento en cuanto a la orientación hacia los padres para derivar a un servicio de atención temprana, ante lo cual los padres proponen que debiese existir un mayor número de profesionales ligados a esta área, cuyo objetivo debiese ser un trabajo preventivo o asistencial en la que se pretenda potenciar al máximo las capacidades del desarrollo y además detectar los signos de alerta de los hitos del desarrollo infantil y en este caso los signos de alerta del trastorno de déficit atencional e hiperactividad.

Un tema que también destaca, está relacionado con las escasas investigaciones existentes en la ciudad de Punta Arenas sobre la detección temprana en las alteraciones del desarrollo infantil, específicamente sobre el TDAH, pese al aumento en la prevalencia del TDAH en los últimos años en la región y a pesar de ser de conocimiento público que el no realizar un diagnóstico oportuno, genera en los niños alteración en su autoestima, aprendizaje y participación social.

Luego del Decreto 170 de la Ley 20.201 (1998) que incluye al Déficit Atencional con y sin Hiperactividad en el Programa de Integración Escolar dirigido a niños con Necesidades Educativas Especiales y que tiene la finalidad de proteger los derechos de las personas con discapacidad, es que se ha visto un aumento en la prevalencia en la región. Este programa contempla la evaluación y colaboración diagnóstica del TDAH, pero sólo en la etapa escolar. Pese a que esto implica un gran avance, aún queda mucho por hacer en relación a la detección temprana del TDAH, debido a la vital importancia que esto radica en los niños y niñas con este trastorno.

El Programa de Integración Escolar incluye dentro de los profesionales colaboradores a los Terapeutas Ocupacionales, esto se debe a que el Terapeuta Ocupacional dentro de su trabajo preventivo y asistencial, tiene conocimientos acerca de la detección temprana en las alteraciones del desarrollo, específicamente conoce los signos evolutivos del TDAH, 
además, posee el conocimiento necesario para potenciar al máximo las habilidades y capacidades de los niños en su período de desarrollo más importante, desde los 0 a los 7 años. Por otra parte el Terapeuta Ocupacional tiene una mirada holística que le permite entender al niño y en general al ser humano en todas sus dimensiones, incorporando a la escuela, la familia y todos los contextos en los cuales el niño se desenvuelve, interviniendo en ellos para asegurar de esta manera una verdadera inclusión social.

En términos generales, los resultados de la investigación son elocuentes en cuanto a la necesidad de que exista una detección oportuna en los niños con síntomas y signos de un retraso en el desarrollo psicomotor y específicamente en los signos evolutivos del Trastorno de Déficit Atencional e Hiperactividad. Para ello resulta necesario un mayor número de profesionales capacitados en la prevención y promoción de la salud en etapas pre-escolares, para evitar que la sintomatología del TDAH altere el desempeño ocupacional de los niños, también para que los padres reciban la información oportuna para que así el niño pueda recibir un tratamiento apropiado disminuyendo el impacto en su desempeño ocupacional y los trastornos asociados al TDAH.

Durante el proceso de investigación fue posible identificar limitaciones, las que podrían ser consideradas en futuras investigaciones relacionadas en esta línea investigativa, como el desconocer la existencia de verificadores de eficacia en la entrega de información en los diversos servicios de salud y educación, en relación a la detección de signos de alerta del desarrollo infantil. Por otra parte, es una limitación no disponer de estadísticas nacionales y regionales que estén a disposición del conocimiento de profesionales ligados al área de la salud, sobre la cantidad de niños/as diagnosticados con TDAH al año. Esta información permitiría conocer con exactitud cuántos y a qué edad los niños que consultan son diagnosticados con TDAH.

Esta investigación pretender entregar las siguientes recomendaciones dirigidas a la salud y educación regional y el desarrollo de la profesión de Terapia Ocupacional:

\section{Prevención y Promoción de la Salud en Magallanes.}

- Investigación en área Infantil: Es evidente la necesidad de realizar más investigaciones relacionadas al área infantil, ya que se contribuiría a mejorar el estudio científico y de esta manera establecer un contacto con la realidad, mejorando o creando nuevos procedimientos y técnicas que aporten a la ciencia y a la sociedad, como forma de resolver problemas, específicamente los relacionados con la detección temprana de trastornos del desarrollo infantil.

- $\quad$ Salud y Educación: Es indudable la necesidad de que exista un mayor número de profesionales en las redes de Atención Primaria relacionados con la prevención y promoción de la salud, específicamente Terapeutas Ocupacionales, ya que poseen un amplio conocimiento sobre el desarrollo psicomotor del niño, signos de alerta y retraso en las diferentes áreas del menor, y además, están vinculados al tratamiento que se debe otorgar cuando esto ocurre, incorporando en él a todos los que participan en la vida del niño como la familia y escuela, para asegurar de esta forma una verdadera inclusión social. Los profesionales vinculados al desarrollo infantil deben 
estar capacitados acerca del conocimiento del desarrollo psicomotor, esto involucra, profesionales tanto de salud como de educación.

Terapia Ocupacional en Magallanes.

- Salud y Educación: Es indudable la necesidad de incrementar el número de Terapeutas Ocupacionales en los establecimientos educacionales y de la salud, ya que podría asegurar el aumento en la detección temprana, oportuno diagnóstico del TDAH y de otros trastornos del desarrollo infantil y entrega de apoyo e información adecuada hacia la familia.

"El holismo orienta a los terapeutas a reconocer cómo se interrelacionan los fenómenos. Nos da una visión del mundo similar a una vasta red de componentes en la cual las partes están incorporadas a un todo" (Polonio, L.

Durante, M. Noya, A. 2001)

- Inclusión Social: El Terapeuta Ocupacional al tener una mirada holística del ser humano, considera no sólo al niño dentro de su tratamiento, sino que además considera a los diferentes contextos donde el niño se desenvuelve, facilitando en todo momento el óptimo desempeño ocupacional del menor, y como fin último, su inclusión social.

Abordaje Familiar.

- Es indispensable que los padres reciban una adecuada información sobre el desarrollo infantil y de las alteraciones que se producen en edades tempranas, en los controles de salud existentes en los programas nacionales, ya que los padres juegan un rol fundamental como observadores y detectores de posibles signos de alerta dentro del desarrollo del niño.

\section{AGRADECIMIENTOS}

Agradecemos a las madres y padres que colaboraron desinteresadamente en este estudio. 


\section{REFERENCIAS BIBLIOGRÁFICAS}

- Círez, I., Pérez, B. y López, S. (2009). Terapia ocupacional en atención temprana. Revista Electrónica de Terapia Ocupacional Galicia, 6(4), 382-390. Recuperado el 23 de diciembre de 2011, de http://www.revistatog.com/suple/num4/atemprana.pdf

- Figueiras, A. C., \& Program on Communicable Diseases (Pan American Health Organization). (2006). Manual para la vigilancia del desarrollo infantil en el contexto de AIEPI. Washington, DC: Organización Panamericana de la Salud.

- Goetz, J. P. \& Lecompte, M. D. (1988). Etnografía y diseño cualitativo en investigación educativa. Madrid: Ediciones Morata.

- Grath, L. (2009). El trastorno por déficit de atención (ADD-ADHD). Buenos Aires: Médica Panamericana,

- Kofi, A. (2005). Un concepto más amplio de la libertad: Desarrollo, seguridad y derechos humanos para todos. Informe del Secretario General de las Naciones Unidas. Recuperado el 2 de septiembre de 2011, de http://www.un.org/spanish/largerfreedom/summary.html

- La búsqueda de significados. Barcelona: Paidós.

- Lavados P., Gómez V., Sawada M., Chomalí M., Álvarez M. (2003). Diagnósticos neurológicos en la atención primaria de salud en Santiago, Chile. Revista Neurológica, $36(6), 518-522$.

- Ley $\mathrm{N}^{\circ} 20.201$ modifica el decreto con fuerza de ley $\mathrm{N}^{\circ} 2$, de 1998 , sobre subvenciones a establecimientos educacionales y otros cuerpos legales (2007, 31 de julio). Biblioteca del Congreso Nacional de Chile. Recuperado Octubre 24, 2011, de www.bcn.cl/histley/lfs/hdl-20201/HL20201.pdf

- López-Ibor, A. J. J., Valdés, M. M., \& American Psychiatric Association. (2002). DSM-IV TR: Manual diagnóstico y estadístico de los trastornos mentales . Barcelona: Masson.

- Polonio, L. B., Durante, M. P. \& Noya, A. B. (2001).Conceptos fundamentales de terapia ocupacional. Madrid: Panamericana.

- Taylor, S. J. \& Bogdan, R. (1987). Introducción a los métodos cualitativos de investigación:

- UNICEF (2006). Manual Escuelas Amigas de la Infancia. Recuperado el 15 de noviembre de 2011, de www.unicef.org/spanish/publications/files/Child Friendly Schools Manual SP 0528 2009.pdf

- Vaquerizo-Madrid, J. (2005).Hiperactividad en el niño preescolar: Descripción clínica. Revista de Neurología, 40(1), 25-32. Recuperado el 11 de noviembre de 2011, de http://www.neurologia.com/pdf/Web/40S1/sS1S025.pdf 